\title{
AIP $\mid$ Review of
}

\section{PointFocus XRay Monochromators for Low Angle Diffraction}

Jesse W. M. DuMond

Citation: Review of Scientific Instruments 21, 188 (1950); doi: 10.1063/1.1745527

View online: http://dx.doi.org/10.1063/1.1745527

View Table of Contents: http://scitation.aip.org/content/aip/journal/rsi/21/2?ver=pdfcov

Published by the AIP Publishing

\section{Articles you may be interested in}

Microprobe $x$-ray fluorescence with the use of point-focusing diffractors

Appl. Phys. Lett. 71, 1884 (1997); 10.1063/1.119427

A pointfocusing small angle xray scattering camera using a doubly curved monochromator of a W/Si multilayer

Rev. Sci. Instrum. 67, 688 (1996); 10.1063/1.1146841

PointFocusing TwoCrystal XRay Monochromator for XRay Diffraction

Rev. Sci. Instrum. 28, 1042 (1957); 10.1063/1.1715798

New PointFocusing Monochromator

Rev. Sci. Instrum. 25, 1219 (1954); 10.1063/1.1770990

A Point Focusing XRay Monochromator for the Study of Low Angle Diffraction

J. Appl. Phys. 23, 854 (1952); 10.1063/1.1702319

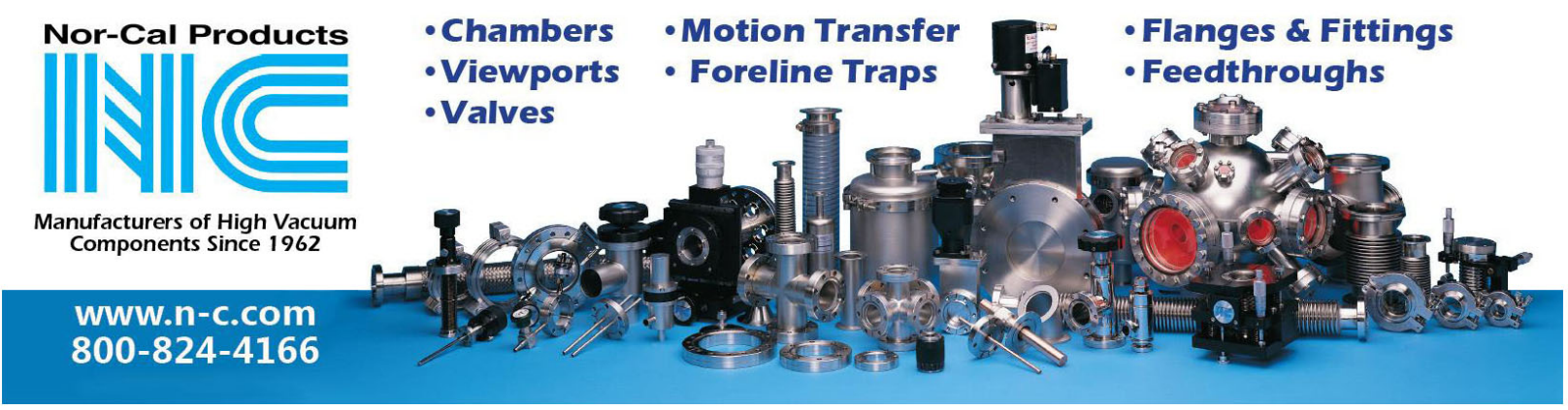




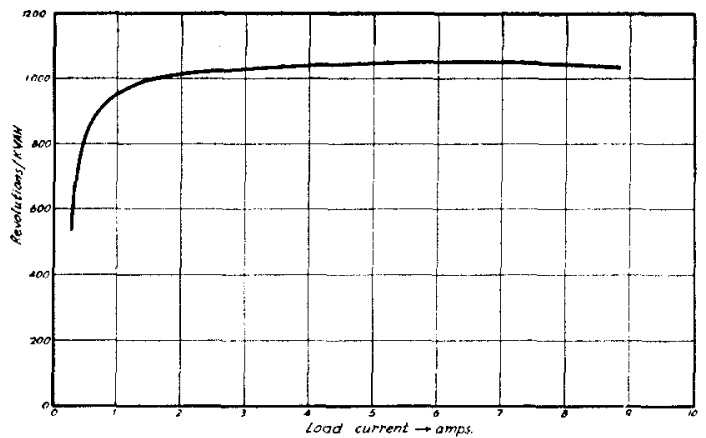

FIG. 4. Meter characteristics at 220 volts a.c.

smoothing, across any one pair of line conductors. Due to the differences in the line currents, three separate current circuits had to be used, each supplying one-third of a triple winding occupying symmetrically the space provided for the two current coils of the d.c. meter.

Regarding the stability of the KVAH-meter described, one can object to the use of metal rectifiers. They suffer, as it is well known, from a resistance variation due to aging. This would result in a slow variation of the meter constant with time. However, modern metal rectifiers seem to maintain their characteristics over long periods without any appreciable variation. Another question arises in connection with the considerable temperature coefficient of the rectifiers. As this coefficient is negative, a temperature compensation of the total circuit seems practicable.

In presenting the above results, the author is not suggesting a device ready for practical use. However, a further investigation as regards the stability of its performance, which seems to be a question of metal rectifiers construction, may lead to a possibly cheap solution of the KVAH-meter problem.

Acknowledgment.- The author expresses his best thanks to Messrs. Ferranti Ltd., Hollinwood, England, for valuable information.

${ }^{1} \mathrm{~A} \mathrm{KVAH-meter} \mathrm{using} \mathrm{rectification} \mathrm{is} \mathrm{described} \mathrm{in} \mathrm{the} \mathrm{British} \mathrm{Patent}$ Specification 357,347 . Its principle is substantially different. due to the lack of a smoothing device and of an air-gap in the transformer core. In order to reduce power factor influence, the single-phase meter described in the patent should have at least two potential circuits, out of phase.

2The design of a device transforming an a.c. into a proportional d.c. has been the subject for the Mech. and Elec. Engineering degree thesis, of Miss. D. Kokkinou.

\section{Simple Low Power Electronic Relay}

William P. RatchFord aNd M. L. FEIN Eastern Regional Research Laboratory, Philadelphia, Pennsylvania* November 28, 1949

$\mathrm{T}$ HE recent note of Linnel and Haendler ${ }^{1}$ describing a simple thermostat relay in which an $F G 57$ thyratron controlled several hundred watts prompts us to describe a similar inexpensive low power circuit (Fig. 1), which we have used for about a year with a precision manostat. ${ }^{2}$ This relay used a 2050 thyratron.

In order that the signal grid shall remain in control when the anode is positive, terminal (2) of the secondary must be $180^{\circ}$ out of phase with terminal (1) of the primary. This is conveniently determined by trial after the relay has been assembled. If the tube fires continuously, the leads from the secondary should be reversed.

The relay has satisfactorily operated the air inlet valve of the manostat; $L$ is the 750 -ohm solenoid of the valve. When an increase in pressure closes the regulator contacts, the filament voltage is applied across $R_{1}$ and is thus impressed on the signal grid out of phase with the anode voltage. The tube does not fire, and the air inlet closes. When the pressure falls and opens the contacts, the signal grid is no longer biased to cut-off and loses control. The tube fires and energizes the solenoid, thus opening the air inlet, and the cycle repeats. $R_{2}$ limits grid current.

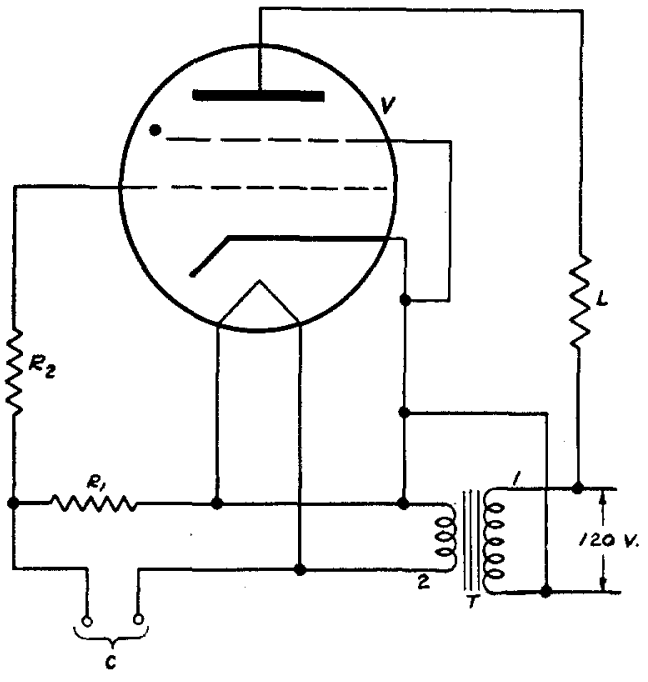

F1G. 1. Low power relay. $T=6.3$ volt a.c. 1 amp. $R_{1}-R_{2}=1$ megohm. 1 watt. $V=2050$ thyratron. $L=750-800 \mathrm{ohm}$ solenoid. $C=$ connection to regulator.

Although suitable for controlling only low power (10 watts or less), the relay has replaced a commercial unit costing five times as much

We wish to thank Dr. C. R. Eddy of this Laboratory for helpful suggestions.

* One of the Laboratories of the Bureau of Agricultural and Industrial Chemistry, Agricultural Research Administration, United States Department of Agriculture.

1 R. H. Linnel and H. M. Haendler, Rev. Sci. Inst. 20, 364 (1949).

2 W. P. Ratchford and M. L. Fein (to be published).

\section{Point-Focus X-Ray Monochromators for Low Angle Diffraction}

Jesse W. M. DUMOND

California Institute of Technology, Pasadena, California November 21, 1949

$\mathrm{G}$ UINIER $^{1}$ has applied the principle of the reflection type curved crystal focusing $x$-ray monochromator ${ }^{2}$ to improve both intensity and angular resolution in low angle $\mathrm{x}$-ray diffraction studies. In Guinier's method, a thin sample is placed in the convergent monochromatic $x$-ray beam and the low angle diffrac-

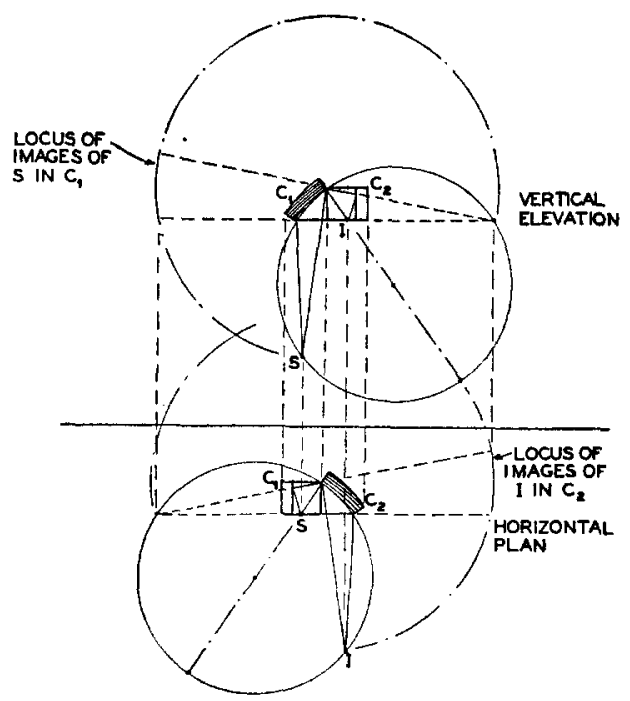

Fig. 1. Point-focus $\mathrm{x}$-ray monochromator. 
tion pattern is formed as a diffuse distribution symmetrically disposed on either side of the central primary line focused by the crystal.

An $x$-ray monochromator capable of forming a beam converging to a point focus would have the following important advantages. (1) The scattered intensity incident in the focal plane would be increased (relative to the line focus monochromator) in the ratio of the feasible line height to the line width, perhaps several hundred fold. (2) The awkward and complicated analytical procedure $^{3}$ for transforming the line focus diffraction pattern distribution into the point focus distribution would be completely avoided. (3) In the case of the two crystal monochromator described below, the residual factor limiting the sensitivity of the method, namely the background fog from incoherent scattering of the continuous $\mathrm{x}$-ray spectrum incident on the first crystal, would be greatly reduced because practically only monochromatic radiation reaches the second crystal.

The type illustrated in Fig. 1 can be made of two monocrystalline quartz lamina cut to a cylindrical shape in the unstressed state, the concave surface being of radius $R$. The lamina should be cut so that the $(310)$ planes $(d \cong 1 A)$ are parallel to the plane tangent to the cylinder at one end of the cylindrical arc. The lamina must then be elastically bent in a precisely profiled holder so that the surface which had radius $R$ now has a radius $R / 2$. This will fulfill the condition for monochromatization and exact focusing for all points on the cylindrical crystal arc. The full line circles are the loci of real focal points (both source and image) for each crystal. The foci shown are approximately those for $\mathrm{Cu}-\mathrm{K}$ radiation. The dot-and-dash circles are the loci on which lie the images of each focal point mirrored in the different (310) planes along the bent crystal arc. The generators of the two cylindrical crystal surfaces are mutually at right angles. Thus, each crystal causes the final beam to converge in one of the two dimensions normal to its direction of propagation. Figure 1 shows the disposition of the components in both horizontal and vertical projection. The crystals are disposed so that the pertinent short arc of the dot-and-dash circle, on which the image of $S$ (or $I$ ) lies, is in each case projected upon the plane of the other view approximately as a point coincident with $I$ (or $S$ ). The (thin) scattering sample is to be placed near the second crystal in the final doubly converging beam, and for best focusing, it should coincide nearly with the polar region of the surface of a sphere whose opposite pole is at the final focus of the beam.

In the second type of point-focusing monochromator illustrated in Fig. 2, use is made of a mica crystal which is bent by cementing it (under pressure with a rubber cushion) to the carefully figured concave surface of a metal block having two different principal

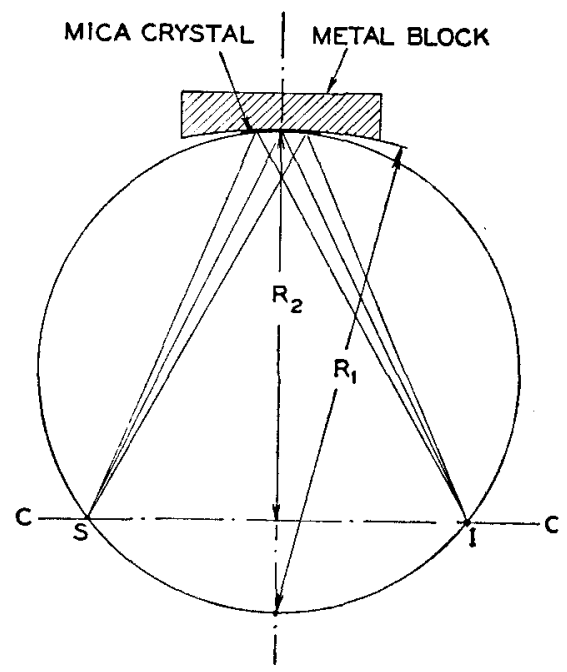

F1G. 2, Point-focusing $x$-ray monochromator. radii of curvature, $R_{1}$ and $R_{2}$. The dot-and-dash line $C C$ is the axis of rotation of the lathe in which such a concave surface is generated. It is easy to show that $R_{2} / R_{1}=\sin ^{2} \theta$ where $\theta$ is the Bragg angle. The figure shows the approximate geometry for monochromatizing fluorine $\mathrm{K}$ radiation from a fluoride target. The permissible crystal arc of radius $R_{1}$ is limited by a well-known aberration ${ }^{4}$ but for the production of a point focus, there is no limit whatever to the extent of the arc of radius $R_{2}$ (about the axis $C C$ ). The arc may be a complete circle if convenient. To insure good homogeneity of scattering angle, however, it would be preferable to use the complete circle design only for a much shorter wave-length and smaller Bragg angle.

The circularly symmetrical low angle diffraction patterns thus obtained could be studied very conveniently by direct G-M counter methods. To do this, an opaque concentric circular stop of variable radius $r$ would be inserted in the pattern and the counter would measure the intensity associated with that part of the pattern outside the radius $r$.

1 A. Guinier, Ann. de physique 12, 161 (1939).

2 J. W. M. DuMond and H. A. Kirkpatrick, Rev. Sci. Inst. 1, 90 (1930), see Fig. 2.

3 J. W. M. DuMond, Phys. Rev. 72, 83 (1947)

4 J. W. M. DuMond, Rev. Sci. Inst. 18, 629 (1947), see Fig. 3.

\section{Ultrasonic Tissue Disintegrator}

\author{
F. F. BIRD AND K. S. LION \\ Laboratory of Applied Biophysics, Department of Biology. \\ Massachusetts Institute of Technology. \\ Cambridge, Massachusetts \\ December 8, 1949
}

7 HE ultrasonic disintegration of organic matter for electron microscopic and other investigations requires a high concentration of energy in the treated substance. This energy is frequently conveyed from a crystal transducer through an oil bath

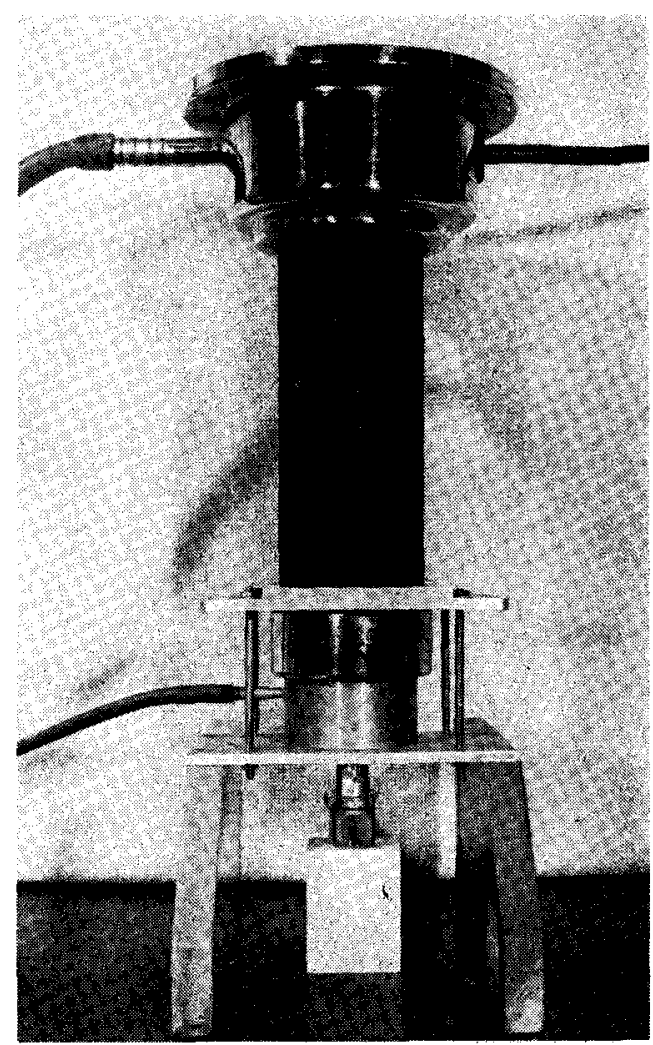

FIG. 1. 